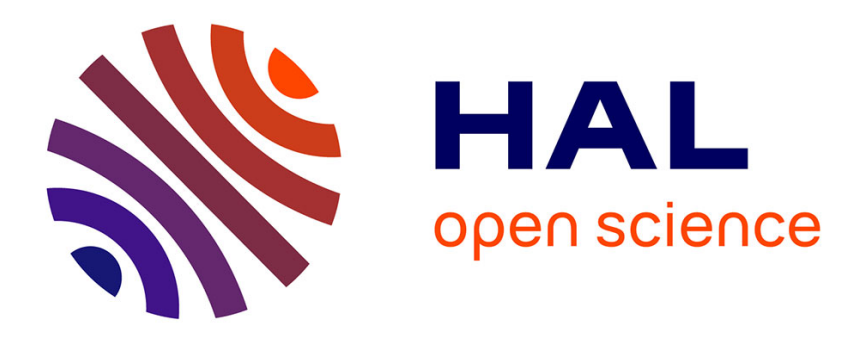

\title{
Etude hydrostatique d'un système de croissance de ruban de silicium
}

\author{
M. Hamidi
}

\section{To cite this version:}

M. Hamidi. Etude hydrostatique d'un système de croissance de ruban de silicium. Revue de Physique Appliquée, 1983, 18 (11), pp.683-689. 10.1051/rphysap:019830018011068300 — jpa-00245131

\section{HAL Id: jpa-00245131 https://hal.science/jpa-00245131}

Submitted on 1 Jan 1983

HAL is a multi-disciplinary open access archive for the deposit and dissemination of scientific research documents, whether they are published or not. The documents may come from teaching and research institutions in France or abroad, or from public or private research centers.
L'archive ouverte pluridisciplinaire HAL, est destinée au dépôt et à la diffusion de documents scientifiques de niveau recherche, publiés ou non, émanant des établissements d'enseignement et de recherche français ou étrangers, des laboratoires publics ou privés. 


\title{
Etude hydrostatique d'un système de croissance de ruban de silicium
}

\author{
M. Hamidi \\ Equipe de Technologie de la Croissance Cristalline, C.N.R.S., 1, place A. Briand, 92195 Meudon Cedex, France
} (Reçu le 15 avril 1983, révisé le 13 juillet, accepté le 21 juillet 1983)

\begin{abstract}
Résumé. - A partir de la détermination de la forme de la zone liquide les possibilités du procédé de tirage du ruban à partir d'un cylindre en rotation ont été étudiées. Nous avons établi les relations qui existent entre les différents paramètres du système et l'épaisseur du ruban tiré. La stabilité du régime permanent de croissance a été étudiée et nous avons montré qu'après une perturbation quelconque, pouvait exister une évolution dans un sens tendant à ramener le système vers un nouvel état d'équilibre.
\end{abstract}

\begin{abstract}
In the development of the silicon ribbon pulling method from a turning cylinder, the equilibrium shape of the melted floating zone has been investigated in relation with the geometric characteristics of the pulled ribbon. The right position and the thickness of the ribbon have been determined versus different parameters of the system. We have studied the stability of the growth of a ribbon with constant thickness; we show that after slight perturbations, the system tends to move toward a new equilibrium state.
\end{abstract}

\section{Introduction.}

Le procédé de tirage de ruban à partir d'un cylindre tournant [1] a pour objet l'élaboration en continu de rubans minces de silicium cristallin de faible coût. Par son principe, ce procédé se rattache à l'ensemble des techniques de croissance de cristaux de forme imposée par capillarité telles que les techniques E.F.G. [2], Stepanov [3], WEB [4], E.S.P. [5], R.T.R. [6], H.R.G. [7], ou autres. La croissance se fait par tirage, à partir d'une "zone flottante" de matériau fondu maintenu au contact du cristal par les forces de tension superficielle, et la forme du cristal élaboré est contrôlée en imposant la forme de cette zone liquide au voisinage du front de cristallisation. Des études expérimentales ont déjà été entreprises [16].

Nous savons qu'un cristal croît à partir de sa phase liquide de telle manière qu'à l'interface de cristallisation la surface libre du liquide fait avec celle du solide un angle déterminé $\delta$, appelé angle de croissance [8-14]; $\delta$ dépend du matériau et de son orientation cristalline et s'explique par la condition d'équilibre thermodynamique des énergies interfaciales des trois phases solide, liquide et gazeuse [8-10]. Des valeurs comprises entre $8^{\circ}$ et $20^{\circ}$ [11-14] ont été reportées pour l'angle de croissance du silicium cristallin.

La croissance d'un cristal ne se fait à section constante que si en tout point de la ligne frontière solideliquide-gaz, l'angle $\theta$ entre la surface libre du liquide et la direction de croissance est constamment égal à $\delta$.
Dans le présent article, nous nous proposons d'étudier les conditions d'un tel équilibre dans le cas du procédé ci-dessus et les relations qu'elles impliquent entre les différents paramètres du système et ceux du ruban élaboré.

\section{Principe du procédé.}

Le principe du procédé de tirage étudié est représenté schématiquement sur la figure 1. A l'aide d'un moyen de chauffage approprié, une zone fondue étroite est formée le long d'une génératrice d'un cylindre de silicium polycristallin en rotation. Un germe monocristallin est alors amené au contract de la zone liquide et après l'établissement de l'équilibre thermique, lente-

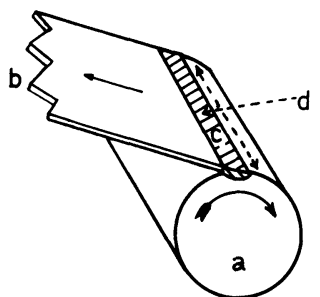

(A)

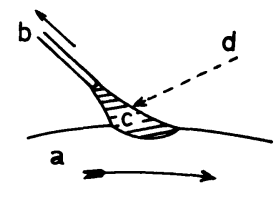

(B)
Fig. 1. - Principe de la méthode : (A) et (B) : a, cylindre en rotation, $b$, ruban tiré, c, zone flottante, $d$, moyen de chauffage.

[Principle of the method : (A) and (B) : a, turning cylinder ; $\mathrm{b}$, pulled ribbon; c, floating zone; $\mathrm{d}$, heating beam.] 
ment éloigné du cylindre dans une direction perpendiculaire à l'axe de rotation de ce dernier. L'alimentation continue de la zone liquide est assurée par la rotation du cylindre; la réduction progressive du diamètre du cylindre par suite du prélèvement de matière au niveau de la zone liquide est compensée par un mouvement de translation verticale de l'axe de rotation de façon à ce que la zone fondue se trouve toujours au même niveau. La puissance $\pi$ et la répartition spatiale de l'énergie de chauffage, la vitesse de rotation $\omega \mathrm{du}$ cylindre, la vitesse de tirage $v_{\mathrm{r}}$ du ruban, ainsi que l'angle $\alpha$ que fait la direction de tirage avec la verticale, constituent des paramètres sur lesquels on peut agir indépendamment, dans les limites permises par l'équilibre général du système.

En fait, l'étude de l'équilibre thermique [15-17] a montré l'existence d'une vitesse limite de rotation $\omega_{1}$ et d'une vitesse limite de tirage $v_{1}$ correspondant aux seuils au-delà desquels l'extraction de la chaleur produite par la solidification du silicium fondu à l'interface solide-liquide n'est plus possible. $\omega_{1}$ est une fonction décroissante du rayon $R$ du cylindre et de la puissance $\pi$; typiquement elle est de l'ordre du dixième de tour par minute. Quant à la vitesse $v_{1}$, elle dépend essentiellement de l'épaisseur $e_{\mathrm{r}}$ du ruban, elle est de l'ordre de $5 \mathrm{~cm} / \mathrm{min}$. pour un ruban de $500 \mu$ d'épaisseur.

\section{Equilibre hydrostatique de la zone fondue.}

3.1 LA LOI DE LAPLACE. - La forme d'équilibre de la surface libre de la zone fondue est régie par la loi de Laplace qui veut qu'à chaque point $M$ de l'interface liquide-gaz, on ait :

$$
P_{\mathrm{e}}-P_{\mathrm{i}}=2 \sigma C
$$

où $P_{\mathrm{e}}-P_{\mathrm{i}}$ est la différence de pression entre l'extérieur et l'intérieur du liquide au point $\mathbf{M}, \sigma$ la tension superficielle du liquide et $C$ la courbure moyenne de l'interface au point $\mathrm{M}$. Si on néglige les mouvements au sein du liquide, et en choisissant les axes de la figure 2 , en un point de cote $z$ :

$$
P_{\mathrm{e}}(z)-P_{\mathrm{i}}(z)=P_{\mathrm{e}}(0)-P_{\mathrm{i}}(0)-\rho g z=\Delta P(0)-\rho g z
$$

( $\rho$ étant la masse volumique du liquide et $g$ l'accélération de la pesanteur).

Notons que dans le procédé de tirage étudié, le liquide étant confiné dans un volume fermé et ne communiquant pas avec un réservoir, $\Delta P(0)$ n'est pas connu a priori et doit être déterminé à partir des autres données de la zone liquide.

Nous supposerons que le ruban est infiniment large de façon à nous ramener à une géométrie à deux dimensions (Fig. 2). L'équation 1 s'écrit alors :

$$
\sigma y_{\mathrm{d}}^{\prime \prime}\left(1+y_{\mathrm{d}}^{\prime 2}\right)^{-3 / 2}=\Delta P(0)-\rho g z
$$

pour un point $\left(y_{\mathrm{d}}, z\right)$ de la partie droite $\mathbf{M}_{2} \mathbf{M}_{3}$ de la

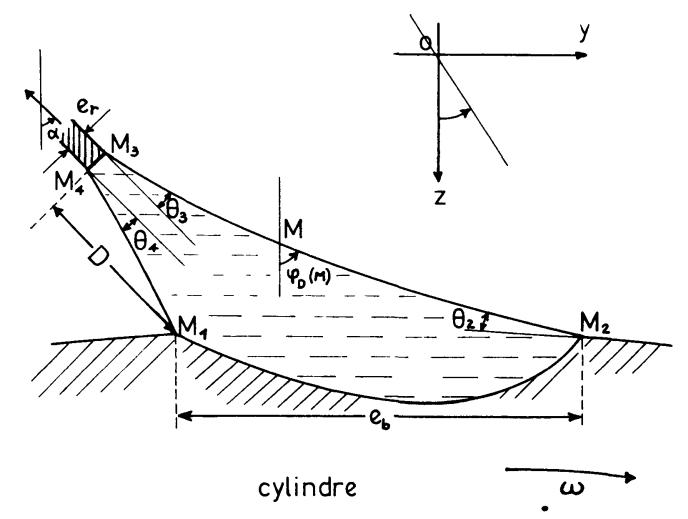

Fig. 2. - Coupe schématique de la zone flottante. Le système d'axes utilisé dans nos calculs est représenté en haut à droite.

[Schematic cross-section of the floating zone.]

surface du liquide, et :

$$
-\sigma y_{\mathbf{g}}^{\prime \prime}\left(1+y_{\mathbf{g}}^{\prime 2}\right)^{-3 / 2}=\Delta P(0)-\rho g z
$$

pour un point $\left(y_{\mathrm{g}}, z\right)$ du profil $\mathbf{M}_{1} \mathbf{M}_{4}$.

Une première intégration nous donne :

$\sin \phi_{\mathrm{d}}(z)=\sin \phi_{3}+\frac{\Delta P(0)}{\sigma}\left(z-z_{3}\right)-\frac{\rho g}{2 \sigma}\left(z^{2}-z_{3}^{2}\right)$

$\sin \phi_{g}(z)=\sin \phi_{4}-\frac{\Delta P(0)}{\sigma}\left(z-z_{4}\right)+\frac{\rho g}{2 \sigma}\left(z^{2}-z_{4}^{2}\right)$

où $\phi_{\mathrm{d}}\left(\right.$ resp. $\phi_{\mathrm{g}}$ ) est l'angle que fait avec la verticale la tangente à la courbe $\mathbf{M}_{2} \mathbf{M}_{3}$ (resp. $\mathbf{M}_{1} \mathbf{M}_{4}$ ) au point de cote $z ; \phi_{3}=\phi_{\mathrm{d}}\left(z_{3}\right)$ et $\phi_{4}=\phi_{\mathrm{g}}\left(z_{4}\right) ; z_{3}$ et $z_{4}$ étant respectivement les cotes des points $\mathbf{M}_{3}\left(y_{3}, z_{3}\right)$ et $\mathbf{M}_{4}\left(y_{4}, z_{4}\right)$.

En intégrant une seconde fois :

$$
\begin{aligned}
& y_{\mathrm{d}}(z)=y_{3}+\int_{z_{3}}^{z}\left(\frac{1}{\sin ^{2} \phi_{\mathrm{d}}(t)}-1\right)^{-1 / 2} \mathrm{~d} t \\
& y_{\mathrm{g}}(z)=y_{4}-\int_{z_{4}}^{z}\left(\frac{1}{\sin ^{2} \phi_{\mathrm{g}}(t)}-1\right)^{-1 / 2} \mathrm{~d} t
\end{aligned}
$$

t représentant la cote d'un point du profil $\mathbf{M}_{2} \mathbf{M}_{3}$ (resp. $\mathbf{M}_{1} \mathbf{M}_{4}$ ) compris entre $\mathbf{M}_{3}$ (resp. $\mathbf{M}_{4}$ ) et le point de cote $z$.

3.2 Conditions DU RÉGime PERMANENT. - Dans la pratique, nous nous intéresserons surtout au régime que nous appellerons " régime permanent de croissance " qui correspond à la situation où les caractéristiques géométriques de la zone liquide ne varient pas au cours de la croissance.

Ceci implique en particulier que les dimensions du 
ruban élaboré restent constantes ou, comme nous l'avons vu précédemment, que :

$$
\theta_{4}=\theta_{3}=\delta
$$

où $\theta_{3}=\phi_{3}-\alpha$ (resp. $\left.\theta_{4}=\phi_{4}+\alpha\right)$ est l'angle que fait la tangente à la surface du liquide au point $\mathbf{M}_{3}$ (resp. $\mathbf{M}_{4}$ ) avec la direction de la croissance et $\delta$ l'angle de croissance du silicium. Donc :

$$
\begin{aligned}
& \phi_{3}=\delta+\alpha \\
& \phi_{4}=\delta-\alpha .
\end{aligned}
$$

Au niveau du cylindre, c'est surtout le point $\mathbf{M}_{2}$ (Fig. 2) qui peut se déplacer par suite de la recristallisation d'une partie du liquide contenu dans la zone flottante. Pour que la position du point $\mathbf{M}_{2}$ reste constante, il faudra :

$$
\theta_{2}=\delta
$$

$\theta_{2}=\frac{\pi}{2}-\phi_{2}-\operatorname{arctg}\left(e_{\mathrm{b}} / R\right)$ étant l'angle que fait la surface du liquide en $\mathbf{M}_{2}$ avec la surface du cylindre ( $R$ rayon du cylindre, $e_{\mathrm{b}}=\left|\mathrm{M}_{1} \mathbf{M}_{2}\right|$, épaisseur de la base de la zone liquide)

$$
\phi_{2}=\frac{\pi}{2}-\delta-\operatorname{arctg}\left(\frac{e_{\mathrm{b}}}{R}\right)
$$

Enfin, la conservation de la matière s'écrit compte tenu de la constance du volume total du liquide :

$$
e_{\mathrm{r}} v_{\mathrm{r}}=\Delta r v_{\mathrm{c}}
$$

où $v_{\mathrm{r}}$ est la vitesse de tirage du ruban, $v_{\mathrm{c}}=\omega R$ la vitesse linéaire d'un point de la surface du cylindre et $\Delta r=r\left(\mathrm{M}_{1}\right)-r\left(\mathrm{M}_{2}\right)$ la réduction de rayon du cylindre à chaque tour.

\section{Formes d'équilibre.}

4.1 Cas génÉRAL. - Avec les conditions 5, 6, 7 et 8 nous sommes en mesure de déterminer complètement la forme de la zone liquide en nous donnant les coordonnées des points $\mathbf{M}_{3}$ et $\mathbf{M}_{4}$ et la cote $z_{2}$ du point $\mathbf{M}_{2}$. En effet, à partir des conditions 5 et 7 et en utilisant la relation 3 appliquée au point $\mathrm{M}_{2}$, on calcule $\Delta P(0)$ par :

$$
\Delta P(0)=\sigma \frac{\sin \phi_{2}-\sin \phi_{3}}{z_{2}-z_{3}}+\frac{\rho g}{2 \sigma}\left(z_{2}+z_{3}\right) .
$$

Les relations 4 et $4^{\prime}$ nous permettent alors de déterminer les profils $\mathbf{M}_{1} \mathbf{M}_{4}$ et $\mathbf{M}_{2} \mathbf{M}_{3}$.

Dans la pratique, $\mathbf{M}_{3}$ et $\mathbf{M}_{4}$ ne sont pas connus a priori et on cherche à les déterminer en fonction des autres conditions aux limites. En plus, plutôt que la forme exacte de la zone liquide c'est surtout les relations existant entre les différents paramètres du système qui nous intéressent. En particulier l'épaisseur $e_{\mathrm{r}}=\left|\mathrm{M}_{3} \mathrm{M}_{4}\right|$ du ruban susceptible d'être tiré en régime permanent est d'un grand intérêt pratique. En tenant compte de tous les paramètres indépendants qui influent sur la forme de la zone fondue $e_{\mathrm{r}}$ peut être déterminé par une relation de la forme :

$$
e_{\mathrm{r}}=f\left(D, e_{\mathrm{b}}, \alpha, v_{\mathrm{r}} / v_{\mathrm{c}}, e_{\mathrm{b}} / R\right)
$$

où $D$ est la distance entre le point $M_{2}$ sur le cylindre et l'interface de solidification $\mathbf{M}_{3} \mathbf{M}_{4}$ que l'on supposera rectiligne et perpendiculaire à la direction de croissance (Fig. 2)

$$
\begin{gathered}
D=\left(z_{2}-z_{3}\right) \cos \alpha+\left(y_{1}-y_{3}\right) \sin \alpha \\
z_{4}-z_{3}=\left(y_{3}-y_{4}\right) \operatorname{tg} \alpha \\
e_{\mathrm{r}} \cos \alpha=y_{3}-y_{4} .
\end{gathered}
$$

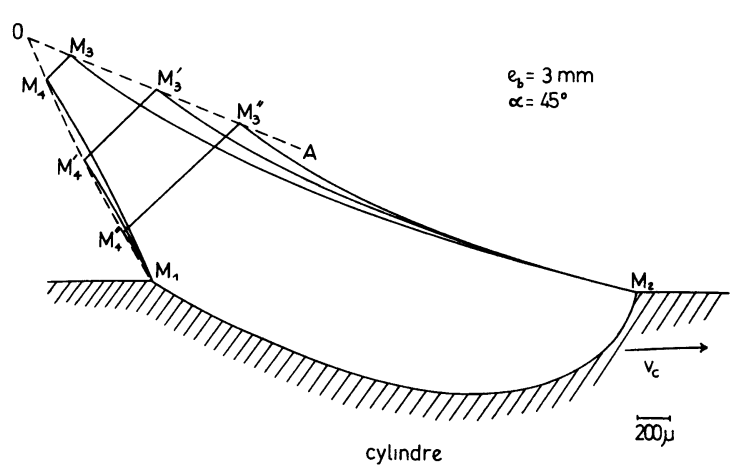

Fig. 3. - Formes calculées de la zone flottante correspondant au régime permanent de croissance; pour $e_{\mathrm{b}}=3 \mathrm{~mm}, \alpha=45^{\circ}, R \gg e_{\mathrm{b}}, v_{\mathrm{r}} \ll v_{\mathrm{c}}$ et pour trois valeurs différentes de $D$. En pointillé, lieux géométriques des points $M_{3}$ et $M_{4}$ quand $D$ varie.

[Calculated shape of the floating zone for $e_{\mathrm{b}}=3 \mathrm{~mm}$, $\alpha=45^{\circ}, R \gg e_{\mathrm{b}}, v_{\mathrm{r}} \ll v_{\mathrm{c}}$ and for three different values of $D$. Doted lines OA and $\mathrm{OM}_{1}$ represent loci of the points $M_{3}$ and $M_{4}$ when $D$ varies.]

Pour simplifier nous supposerons que le rayon du cylindre est assez grand pour que $e_{\mathrm{b}} / R$ soit négligeable devant $\delta$. La relation 7 nous donne alors :

$$
\phi_{2}=\frac{\pi}{2}-\delta
$$

Et la relation 8 s'écrit :

$$
z_{1}-z_{2}=e_{\mathrm{r}} \frac{v_{\mathrm{r}}}{v_{\mathrm{c}}}
$$

4.2 A faible VITESSE DE TIRAGE. - Pour réduire encore le nombre de paramètres nous nous sommes intéressés dans un premier temps au cas où le rapport des vitesses $v_{\mathrm{r}} / v_{\mathrm{c}}$ est presque nul de façon à ce que :

$$
z_{1}-z_{2}=0 \text {. }
$$

Les caractéristiques de la zone liquide peuvent alors être calculées en fonction des trois paramètres indépendants $\alpha, e_{\mathrm{b}}$ et $D$. A partir du système d'équations 5 , $6,10,11,12,13,14,4$ appliquée au point $\mathbf{M}_{2}$ et $4^{\prime}$ 
appliquée au point $M_{1}$ nous avons calculé les coordonnées des points $M_{3}$ et $M_{4}$.

Les valeurs numériques utilisées ont été les suivantes :

$$
\begin{array}{ll}
\rho=2,50 \mathrm{~g} / \mathrm{cm}^{3} & {[18]} \\
\sigma=720 \mathrm{erg} / \mathrm{cm}^{2} & {[19]} \\
\delta=11^{\circ} & {[11,13,14] .}
\end{array}
$$

Sur la figure 3 la forme de la zone fondue correspondant au régime permanent de croissance est représentée pour $e_{\mathrm{b}}=3 \mathrm{~mm}, \alpha=45^{\circ}$ et pour plusieurs valeurs différentes de $D$. On remarque en particulier que la distance $\left|\mathbf{M}_{3} \mathbf{M}_{4}\right|$ correspondant à l'épaisseur $e_{\mathrm{r}}$ du ruban tiré diminue quand $D$ augmente. Les courbes $\mathrm{OA}$ et $\mathrm{OM}_{1}$ portées en pointillé sur la même figure représentent les lieux géométriques respectifs de $\mathbf{M}_{3}$ et $\mathbf{M}_{4}$.

Sur la figure 4, nous avons porté ces courbes pour plusieurs valeurs de $\alpha$ et pour $e_{\mathrm{b}}=3 \mathrm{~mm}$. On voit sur cette figure que les lieux géométriques des points $\mathbf{M}_{3}$ peuvent être assimilés en première approximation à des segments de droites dont les prolongements passeraient par $\mathbf{M}_{2}$ et qui feraient avec la verticale un angle à peu près égal à $\frac{\pi}{4}+\frac{\alpha}{2}$. Ceci est surtout vrai pour les faibles valeurs de $\alpha$.

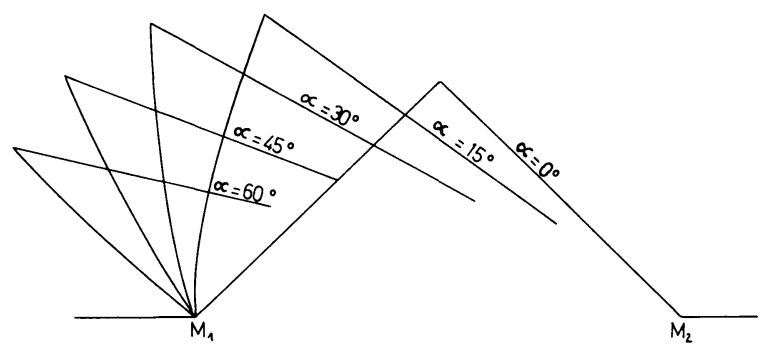

Fig. 4. - Lieux géométriques des points $\mathrm{M}_{3}$ et $\mathrm{M}_{4}$ correspondant au régime permanent de croissance; pour $e_{\mathrm{b}}$ : $3 \mathrm{~mm}, R \gg e_{\mathrm{b}}, v_{\mathrm{r}} \ll v_{\mathrm{c}}$ et pour cinq valeurs différentes de $\alpha$.

[Loci of the points $\mathrm{M}_{3}$ and $\mathrm{M}_{4}$; for $e_{\mathrm{b}}=3 \mathrm{~mm}, R \gg e_{\mathrm{b}}$, $v_{\mathrm{r}} \ll v_{\mathrm{c}}$ and for five different values of $\alpha$.]

Cette propriété se retrouve analytiquement si dans les formules 3 et 4 nous négligeons les termes comprenant le poids volumique $\rho g$ devant les autres termes (ce qui revient à négliger l'influence de la pesanteur sur la forme de la zone liquide). Un calcul simple nous donne alors :

$$
y_{2}=y_{3}+\left(z_{2}-z_{3}\right) \operatorname{tg}\left(\frac{\phi_{3}+\phi_{2}}{2}\right)
$$

qui avec les conditions 5 et 13 s'écrit :

$$
y_{2}=y_{3}+\left(z_{2}-z_{3}\right) \operatorname{tg}\left(\frac{\pi}{4}+\frac{\alpha}{2}\right)
$$

et correspond bien aux droites décrites.
La figure 5 représente les variations de l'épaisseur $e_{\mathrm{r}}$ du ruban en fonction de la distance $D$, pour $e_{\mathrm{b}}=3 \mathrm{~mm}$ et pour plusieurs valeurs différentes de l'angle de tirage. Sur la figure 6, les mêmes courbes sont tracées pour $\alpha$ fixé $\left(\alpha=45^{\circ}\right)$ et pour les valeurs différentes de $e_{\mathrm{b}}$. On constate que dans l'hypothèse

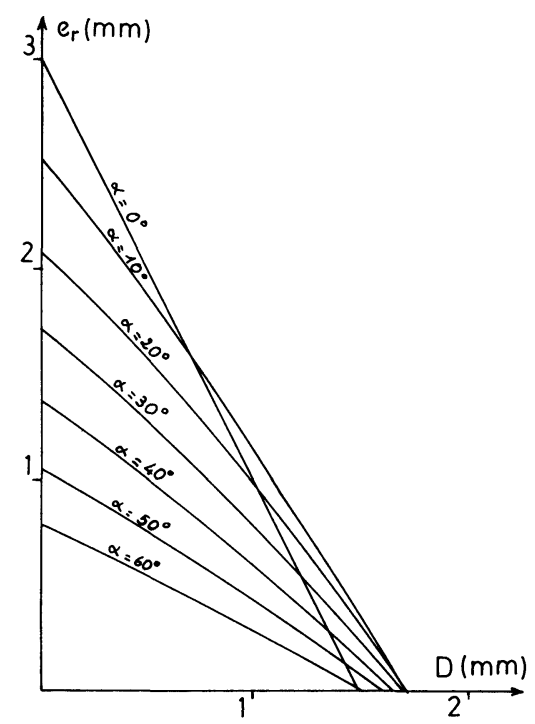

Fig. 5. - Variations de l'épaisseur $e_{\mathrm{r}}$ du ruban en fonction de la distance interface-cylindre $D$; pour $e_{\mathrm{b}}=3 \mathrm{~mm}$, $R \gg e_{\mathrm{b}}, v_{\mathrm{r}} \ll v_{\mathrm{c}}$ et pour différentes valeurs de $\alpha$.

[Variation of the ribbon thickness $e_{\mathrm{r}}$ versus the cylinderinterface distance $D$; for $e_{\mathrm{b}}=3 \mathrm{~mm}, R \gg e_{\mathrm{b}}, \dot{v}_{\mathrm{r}} \ll v_{\mathrm{c}}$ and for different values of $\alpha$.]

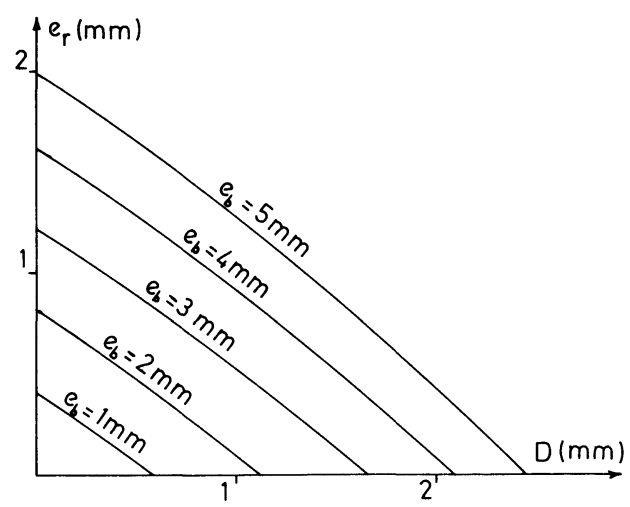

Fig. 6. - Variations de l'épaisseur $e_{\mathrm{r}}$ du ruban en fonction de la distance $D$; pour $\alpha=45^{\circ}, R \gg e_{\mathrm{b}}, v_{\mathrm{r}} \ll v_{\mathrm{c}}$ et pour cinq valeurs différentes de $e_{\mathrm{b}}$.

[Variation of the ribbon thickness $e_{\mathrm{r}}$ versus $D$; for $\alpha=45^{\circ}$, $R \gg e_{\mathrm{b}}, v_{\mathrm{r}} \ll v_{\mathrm{c}}$ and for five different values of $e_{\mathrm{b}}$.]

d'un ruban infiniment large, il n'existe pas de limite inférieure à l'épaisseur du ruban tiré, qui peut être aussi mince qu'on le désire. Nous verrons plus loin qu'il n'en est plus de même si l'on tient compte des effets dus aux bords latéraux de la zone liquide. Des figures 5 
et 6 , nous déduisons de plus, que l'épaisseur du ruban est d'autant plus faible que :

a) l'interface ruban liquide est loin du cylindre,

b) l'angle de tirage est important,

c) l'étendue $e_{\mathrm{b}}$ de la zone fondue sur le cylindre est faible.

4.3 INFLUENCE DE LA VITESSE DE TIRAGE. - Si $v_{\mathrm{r}} / v_{\mathrm{c}}$ n'est plus négligeable on a en plus à tenir compte de la relation 14.

Plutôt que de faire le calcul exact on peut en supposant que la distance $z_{2}-z_{1}$ est faible devant $z_{1}-z_{4}$ se contenter d'un calcul approché.

Pour la même valeur de $z_{3}-z_{2}$ comparons la forme de la zone liquide du cas présent avec celle correspondant au cas où la vitesse de tirage est presque nulle. Le profil de la courbe $\mathbf{M}_{2} \mathbf{M}_{3}$ sera le même dans les deux cas. Sur la figure 7, nous avons représenté les zones liquides correspondantes en superposant les

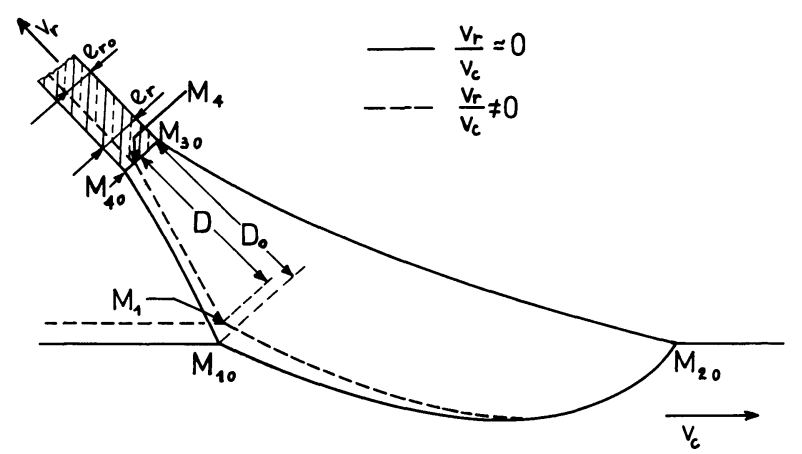

Fig. 7. - Superposition de deux situations différentes de la zone liquide correspondant à deux valeurs distinctes de $v_{\mathrm{r}} / v_{\mathrm{c}}$ mais à la même valeur de $z_{2}-z_{3}$.

[Comparison of the liquid zone between the two cases $v_{\mathrm{r}} / v_{\mathrm{c}} \neq 0$ (doted lines) and $v_{\mathrm{r}} / v_{\mathrm{c}}=0 . z_{2}-z_{3}$ being the same for both cases.]

courbes $\mathbf{M}_{2} \mathbf{M}_{3}$ et $\mathbf{M}_{20} \mathbf{M}_{30}$ (les indices comprenant 0 comme dans $\mathbf{M}_{20} \mathbf{M}_{30}$ correspondant au cas où $v_{\mathrm{r}} / v_{\mathrm{c}} \simeq 0$ ). Le « déplacement » du point $\mathrm{M}_{1}$ par rapport à $\mathbf{M}_{10}$ s'accompagne d'un déplacement de $\mathbf{M}_{40}$ en $\mathbf{M}_{\mathbf{4}}$ et donc d'un changement de $D_{0}$ et de $e_{\mathrm{r}_{0}}$

$$
\begin{gathered}
D=D_{0}-\left(z_{2}-z_{1}\right) \cos \alpha=D_{0}-e_{\mathrm{r}} \frac{v_{\mathrm{r}}}{v_{\mathrm{c}}} \cos \alpha \\
e_{\mathrm{r}}=e_{\mathrm{r}_{0}}-\frac{z_{40}-z_{4}}{\sin \alpha} .
\end{gathered}
$$

La formule $4^{\prime}$ appliquée au point $M_{1}$ nous donne après plusieurs approximations :

$$
y_{1}-y_{4} \simeq y_{10}-y_{40}+\left(z_{40}-z_{4}-z_{10}+z_{1}\right) \operatorname{tg} \phi_{2} \text {. }
$$

Or,

et

$$
y_{1}=y_{10} ; \quad z_{10}=z_{2}
$$

$$
z_{40}-z_{4}=\left(y_{4}-y_{40}\right) \operatorname{tg} \alpha \text {. }
$$

D'où en partant de 16

$$
\left(z_{40}-z_{4}\right)\left(\operatorname{tg} \phi_{2}+\operatorname{tg} \alpha\right) \simeq e_{\mathrm{r}} \frac{v_{\mathrm{r}}}{v_{\mathrm{c}}} \operatorname{tg} \phi_{2}
$$

Et, en comparant avec (15) :

$$
\begin{gathered}
e_{\mathrm{r}} \simeq e_{\mathrm{r}_{0}}\left(1+\frac{\operatorname{tg} \phi_{2}}{\cos \alpha+\sin \alpha \operatorname{tg} \phi_{2}} \frac{v_{\mathrm{r}}}{v_{\mathrm{c}}}\right)^{-1} \\
D \simeq D_{0}-e_{\mathrm{r}_{0}}\left(\frac{v_{\mathrm{c}}}{v_{\mathrm{r}}}+\frac{\operatorname{tg} \phi_{2}}{\cos \alpha+\sin \alpha \operatorname{tg} \phi_{2}}\right)^{-1} .
\end{gathered}
$$

A partir des courbes $e_{\mathrm{r}_{0}}=f\left(D_{0}\right)$ déjà terminées, on peut tracer les courbes $e_{\mathrm{r}}=f_{v_{\mathrm{r}} / v_{\mathrm{c}}}(D)$ calculées pour $v_{\mathrm{r}} / v_{\mathrm{c}}$ donné à l'aide des formules ci-dessus. La figure 8 représente une série de telles courbes pour $\alpha=45^{\circ}$ et $e_{\mathrm{b}}=3 \mathrm{~mm}$ on voit que pour une valeur donnée de $D, e_{\mathrm{r}}$ est d'autant plus faible de $v_{\mathrm{r}} / v_{\mathrm{c}}$ est élevé.

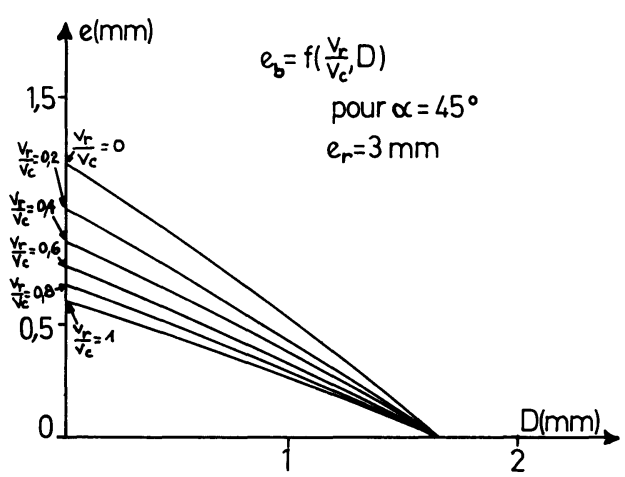

Fig. 8. - Variations de l'épaisseur du ruban en fonction de $D$; pour $\alpha=45^{\circ}, e_{\mathrm{b}}=3 \mathrm{~mm}, R \gg e_{\mathrm{b}}$ et pour différentes valeurs de $v_{\mathrm{r}} / v_{\mathrm{c}}$.

[Variation of the ribbon thickness $e_{\mathrm{r}}$ versus $D$; for $\alpha=45$, $e_{\mathrm{b}}=3 \mathrm{~mm}, R \gg e_{\mathrm{b}}$ and different values of $v_{\mathrm{r}} / v_{\mathrm{c}}$.]

\section{Stabilité du régime permanent.}

La question de la stabilité de la croissance est d'un grand intérêt pratique. Supposons que par suite d'une perturbation quelconque le système soit écarté des conditions du régime permanent qui sont, rappelonsles :

et

$$
\theta_{2}=\theta_{3}=\theta_{4}=\delta
$$

$$
e_{\mathrm{r}} v_{\mathrm{r}}=\Delta r v_{\mathrm{c}}
$$

Supposons, par exemple, que la valeur de $\theta_{4}$ ait changé et appelons $\theta_{4 \mathrm{p}}$ sa nouvelle valeur. La position du point $\mathbf{M}_{4}$ va alors évoluer au cours de la croissance. Si par exemple, $\theta_{4 \mathrm{p}}$ est inférieur à $\delta, \mathbf{M}_{4}$ va se déplacer vers la droite sur la figure 2 de sorte que l'épaisseur de l'interface diminue. Le déplacement du point $\mathbf{M}_{4}$ induit à son tour une variation de $\theta_{4 p}$, soit vers sa valeur d'équilibre, auquel cas la perturbation sera automa- 
tiquement résorbée, soit vers une valeur encore plus grande, ce qui amplifiera la perturbation.

Sur la figure 9, on a représenté les courbes $e_{\mathrm{r}}=f(D)$ pour $e_{\mathrm{b}}=3 \mathrm{~mm}, \alpha=45^{\circ}, v_{\mathrm{r}} / v_{\mathrm{c}}=0, \theta_{2}=\theta_{3}=\delta$ et pour trois valeurs différentes de $\theta_{4}: \theta_{4}=\delta$, $\theta_{4}<\delta$ et $\theta_{4}>\delta$. L'exemple ci-dessus correspond à un point $\mathrm{S}_{1}$ situé sur la courbe $\theta_{4}<\delta$. Nous avons vu que dans ce cas la croissance du ruban s'accompagne d'une diminution de son épaisseur. Si on suppose que $\theta_{2}, \theta_{3}$ et $D$ ne sont pas affectés par l'évolution de $e_{\mathrm{r}}$ le point représentant le système sur la figure 9 va se déplacer vers le bas selon le trajet indiqué et $\theta_{4}$ va donc croître jusqu'à sa valeur d'équilibre $\delta$ (point $S_{2}$ de la figure 9). La perturbation n'induit pas d'instabilité et la croissance se poursuit dans un nouvel état d'équilibre.

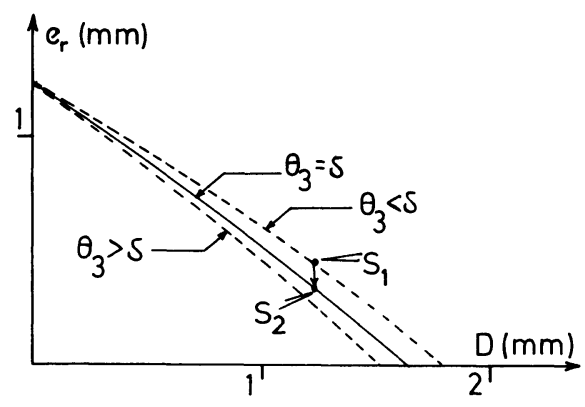

Fig. 9. - Variations de $e_{\mathrm{r}}$ en fonction de $D$; pour $e_{\mathrm{b}}=3 \mathrm{~mm}$, $\alpha=45^{\circ}, v_{\mathrm{r}} \ll v_{\mathrm{c}}, R \gg e_{\mathrm{b}}, \theta_{2}=\theta_{4}=\delta$ et pour trois valeurs différentes de $\theta_{3}: \theta_{3}=\delta, \theta_{3}<\delta$ et $\theta_{3}>\delta$. On voit que si le système est dans un état perturbé (par exemple, l'état $\mathrm{S}_{1}$ correspondant à $\theta_{3}<\delta$ ) son évolution au cours de la croissance $\left(\theta_{3}<\delta \Rightarrow \mathrm{e}_{\mathrm{r}} \downarrow\right)$ tend à le ramener vers un état d'équilibre (point $S_{2}$ de la figure ci-dessus).

[Variation of $e_{\mathrm{r}}$ versus $D$, for three different values of $\theta_{3}$ : $\theta_{3}=\delta, \theta_{3}<\delta$ and $\theta_{3}>\delta$; with $e_{\mathrm{b}}=3 \mathrm{~mm}, \alpha=45^{\circ}$, $v_{\mathrm{r}} \ll v_{\mathrm{c}}, R \gg e_{\mathrm{b}}$ and $\theta_{2}=\theta_{4}=\delta$. When $\theta_{3}=\delta$ the ribbon grows with constant cross-section; if $\theta_{3}<\delta$, for example, (case of the point $S_{1}$ ) the point $M_{3}$ moves during the growth and $e_{\mathrm{r}}$ decreases; we can see above, that the growth system moves then toward a new equilibrium state $S_{2}$.]

Le raisonnement précédent est en fait assez simpliste à plusieurs titres. Il suppose qu'une perturbation quelconque n'agit que sur une seule des conditions d'équilibre à la fois; ce qui est loin d'être vrai dans le cas pratique. Mais surtout elle néglige le fait que le déplacement du point $\mathbf{M}_{4}$ n'entraîne pas seulement la variation de $\theta_{4 \mathrm{p}}$ mais aussi celle de $\theta_{2}$ et $\theta_{3}$ si bien que quand $\theta_{4}$ a rejoint sa valeur d'équilibre $\theta_{4}=\delta$, ce sont $\theta_{2}$ et $\theta_{3}$ qui ne sont plus égaux à $\delta$ et les points $\mathbf{M}_{2}$ et $\mathrm{M}_{3}$ qui évoluent; la variation de l'épaisseur $e_{\mathrm{r}}$ de l'interface perturbe d'autre part la condition de constance du volume total du liquide (8) et induit par des effets thermiques une variation de $D$.

L'étude exacte de la stabilité du régime permanent de croissance n'est possible qu'en tenant compte de tous ces effets et par la détermination de l'évolution temporelle du système perturbé. Elle dépasse le cadre du présent article et sera présenté par ailleurs. Des considérations simplifiées du type déjà exposé nous renseignent cependant assez bien sur les tendances spontanées du système après une perturbation. Nous avons vu que l'évolution du point $\mathbf{M}_{4}$ se fait dans le sens qui tend à ramener $\theta_{4}$ vers sa valeur d'équilibre. Nous pourrions montrer à l'aide de raisonnements similaires qu'il en est de même en ce qui concerne $\theta_{3}$ et $\theta_{2}$ et les points $\mathbf{M}_{3}$ et $\mathbf{M}_{2}$. Quant à la condition 8 , dans le cas où elle n'est pas vérifiée, le volume total du liquide n'est plus constant. Supposons, par exemple, que $e_{\mathrm{r}} v_{\mathrm{r}}<\Delta r v_{\mathrm{c}}$ le volume total du liquide va augmenter au cours de la croissance. Ceci entraîne un accroissement de $\theta_{2}, \theta_{3}$ et $\theta_{4}$, donc une diminution de $\Delta r$ et une augmentation de $e_{\mathrm{r}}$ par suite du déplacement des points $\mathbf{M}_{2}, \mathbf{M}_{3}$ et $\mathbf{M}_{4}$; ces effets vont dans le sens d'un retour vers l'équilibre de l'équation 8 .

\section{Sensibilité de $e_{\mathrm{r}}$ aux variations de $D$.}

$\mathrm{Si}$, lors de la croissance en régime permanent, la valeur de la distance $D$ varie par suite d'une perturbation du système (par ex. perturbation thermique) l'épaisseur du ruban varie en conséquence pour atteindre une nouvelle valeur d'équilibre. Pour avoir un ruban régulier, nous avons intérêt à ce que les variations induites d'épaisseur $\Delta e_{\mathrm{r}}$ soient les plus faibles possibles. Nous définirons la sensibilité $S$ par $S=\Delta e_{\mathrm{r}} / \Delta D$ ou plus exactement par :

$$
S=\left|\frac{\partial e_{\mathrm{r}}}{\partial D}\right|
$$

$S$ correspond à la valeur absolue de la pente de la tangente aux courbes $e_{\mathrm{r}}=f(D)$ des figures 5,6 et 8 . Sur la figure 10, nous avons porté $S$, en fonction de l'angle de tirage pour $e_{\mathrm{b}}=3 \mathrm{~mm}, e_{\mathrm{r}}=0,5 \mathrm{~mm}$ et $v_{\mathrm{r}} / v_{\mathrm{c}}=0$; nous remarquons que $\mathrm{S}$ est d'autant plus faible que $\alpha$ est important.

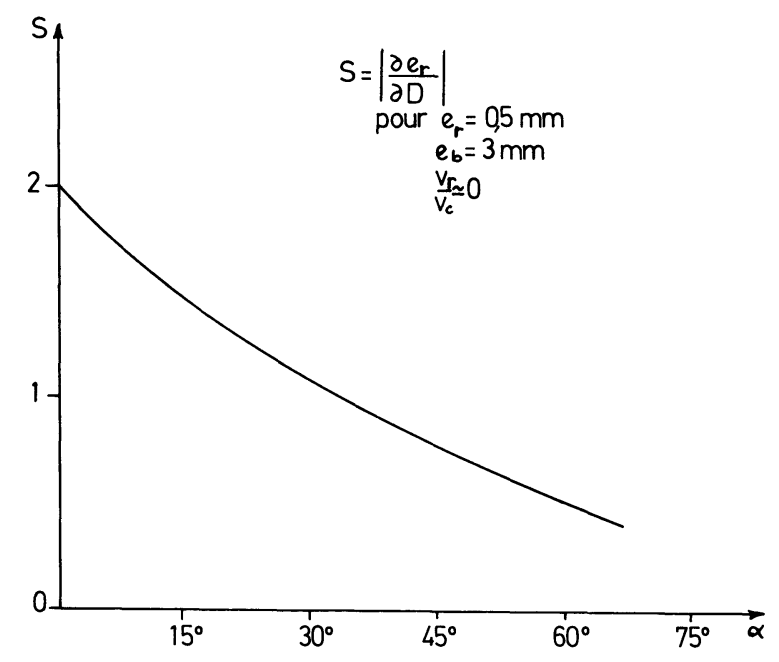

Fig. 10. - Sensibilité $S$ de $e_{\mathrm{r}}$ aux variations de $D$ en fonction de l'angle de tirage $\alpha$; pour $e_{\mathrm{b}}=3 \mathrm{~mm}, e_{\mathrm{r}}=0,5 \mathrm{~mm}$ et $v_{\mathrm{r}} \ll v_{\mathrm{c}}$.

[Variation of the sensibility $S=\left|\partial e_{\mathrm{r}} / \partial D\right|$ versus the pulling angle $\alpha$ for $e_{\mathrm{b}}=3 \mathrm{~mm}, e_{\mathrm{r}}=0.5 \mathrm{~mm}, R \gg e_{\mathrm{b}}$ and $v_{\mathrm{r}} \ll v_{\mathrm{c}}$.] 
7. Problème des extrémités latérales de la zone fondue.

Dans la plupart des procédés de tirage de rubans par capillarité les principales limitations proviennent des effets dus aux bords latéraux de la zone liquide. Dans le cas d'un tirage vertical, le comportement des bords a été étudié, soit par des modélisations à l'aide de formes de révolution $[20,21]$, soit par un calcul numérique en trois dimensions de la géométrie exacte de la surface libre du liquide [22]. Un résultat important peut être déduit de ces études : du fait de la courbure assez forte $\left(1 / R_{1}\right)$ de l'extrémité latérale du ruban $\left(R_{1} \simeq e_{\mathrm{r}} / 2\right)$, une courbure $\left(1 / R_{2}\right)$ de même ordre de grandeur est imposée à la surface du liquide dans une direction perpendiculaire à l'interface de cristallisation. Ceci est nécessaire afin que $2 C=1 / R_{2}-1 / R_{1}$ ait la valeur imposée par l'équation 1 . La hauteur de la zone liquide ne peut alors pas dépasser une valeur limite $D_{1}$ qui est typiquement de l'ordre de $e_{\mathrm{r}} / 2$; ce qui impose en même temps une limite inférieure à la valeur de $e_{\mathrm{r}}$.

En se référant aux courbes de la figure 6 et en supposant que $D_{1}$ reste du même ordre de grandeur pour toutes les valeurs de $\alpha$, on voit que l'épaisseur minimale du ruban est d'autant plus faible que $\alpha$ est important.

Des essais expérimentaux de tirage de ruban par cette méthode ont été relatés dans la référence 16 . Ils ont permis de vérifier certains résultats des études théoriques hydrostatiques et thermiques $[15,16]$. C'est ainsi que, en éliminant artificiellement les effets de bord par l'adjonction de baguettes fines de graphite aux extrémités latérales du germe, quelques rubans d'assez faible épaisseur (environ $500 \mu$ ) ont pu être tirés.

\section{Conclusion.}

L'étude de l'équilibre hydrostatique de la zone liquide nous a montré qu'il est théoriquement possible d'élaborer des rubans de silicium cristallin par le procédé de tirage à partir d'un cylindre tournant. Pour l'obtention d'un ruban mince et régulier, les meilleures conditions expérimentales sont réunies dans le cas où l'étendue de la zone fondue sur le cylindre est faible et où le tirage se fait avec un angle important par rapport à la verticale. Par un raisonnement simplifié, nous avons montré que ce procédé est vraisemblablement stable du point de vue de la croissance régulière d'un ruban d'épaisseur constante. Une étude détaillée de ce dernier point sera publiée prochainement.
[1] Rodot, H., Brevet Fr. no 7911174 (1979).

[2] Ravi, K. V., J. Cryst. Growth 39 (1977) 1.

[3] Antonov, P. I., Nikaronov, S. P. and Tatarchenko, V. A., J. Cryst. Growth 42 (1977) 447.

[4] Seidensticker, R. G., J. Cryst. Growth 39 (1977) 17.

[5] CizeK, T. F., 'HuRd, J. L., Melt growth of silicon sheets by edge-supported pulling, Proceedings of the symposia on electronic and optical properties of polycrystalline or impure semiconductors, St. Louis (1980).

[6] Gurtler, R. W., Baghdadi, A. G., Ellis, R. J. and LESK, I. A., J. Electron. Mat. 7 (1978) 441.

[7] Bleil, C. E., J. Cryst. Growth 5 (1969) 99.

[8] Bradsley, W., Frank, F. C., Green, G. W., Hurle, D. T. J., J. Cryst. Growth 23 (1974) 341.

[9] Voronkov, V. V., Sov. Phys. Crystallogr., 19 (5) (1975) 573

[10] Voronkov, V. V., J. Cryst. Growth 52 (1981) 311.

[11] Surek, T., Chalmers, B., J. Cryst. Growth 29 (1975) 1.

[12] Antonov, P. I., Stepanov, A. V., Bull. Acad. Sci. USSR Phys. Ser. 33 (1969) 1805.

\section{Bibliographie}

[13] Satunkin, G. A., Tatarchenko, V. A. and Shaitanov, V. I., J. Cryst. Growth 50 (1980) 133.

[14] Hamidi, M., Rodot, H., Revue Phys. Appl. 18 (1983) 75 .

[15] Cassagne, Ph., Revue Phys. Appl. 17 (1982) 737.

[16] Cassagne, $\mathrm{Ph}$., Etude théorique et expérimentale d'une nouvelle méthode de croissance de rubans de silicium, Thèse soutenue à l'Université de Paris VII (1982).

[17] Zook, J. D., Schuldt, S. B., J. Cryst. Growth 50 (1980) 51.

[18] Glazov, V. M., Chizheskaya, S. N. and Glagoleva, N. N., Liquid Semiconductors, (ed. Plenum Publishing Corporation).

[19] Davies, G. R., Metall. Rev. 10 (1965) 173

[20] Swartz, J. C., Surek, T., Chalmers, B., J. Electron. Mat. 4 (2) (1975) 255.

[21] Belouet, Ch., Compte Rendu de fin d'étude, D.G.R.S.T. (1978)

[22] Rodot, H., Cassagne, Ph., Hamidi, M., J. Electron. Mat. 10 (1981) 481. 